\title{
Association of Prenatal Maternal Depression and Anxiety Symptoms With Infant White Matter Microstructure
}

Douglas C. Dean III, PhD; Elizabeth M. Planalp, PhD; William Wooten, MS; Steven R. Kecskemeti, PhD; Nagesh Adluru, PhD; Cory K. Schmidt, BA; Corrina Frye, BS; Rasmus M. Birn, PhD; Cory A. Burghy, PhD; Nicole L. Schmidt, MS; Martin A. Styner, PhD; Sarah J. Short, PhD; Ned H. Kalin, MD; H. Hill Goldsmith, PhD; Andrew L. Alexander, PhD; Richard J. Davidson, PhD

IMPORTANCE Maternal depression and anxiety can have deleterious and lifelong consequences on child development. However, many aspects of the association of early brain development with maternal symptoms remain unclear. Understanding the timing of potential neurobiological alterations holds inherent value for the development and evaluation of future therapies and interventions.

OBJECTIVE To examine the association between exposure to prenatal maternal depression and anxiety symptoms and offspring white matter microstructure at 1 month of age.

DESIGN, SETTING, AND PARTICIPANTS This cohort study of 101 mother-infant dyads used a composite of depression and anxiety symptoms measured in mothers during the third trimester of pregnancy and measures of white matter microstructure characterized in the mothers' 1-month offspring using diffusion tensor imaging and neurite orientation dispersion and density imaging performed from October 1, 2014, to November 30, 2016. Magnetic resonance imaging was performed at an academic research facility during natural, nonsedated sleep.

MAIN OUTCOMES AND MEASURES Brain mapping algorithms and statistical models were used to evaluate the association between maternal depression and anxiety and 1-month infant white matter microstructure as measured by diffusion tensor imaging and neurite orientation dispersion and density imaging findings.

RESULTS In the 101 mother-infant dyads (mean [SD] age of mothers, 33.22 [3.99] years; mean age of infants at magnetic resonance imaging, 33.07 days [range, 18-50 days]; 92 white mothers [91.1\%]; 53 male infants [52.5\%]), lower 1-month white matter microstructure (decreased neurite density and increased mean, radial, and axial diffusivity) was associated in right frontal white matter microstructure with higher prenatal maternal symptoms of depression and anxiety. Significant sex $\times$ symptom interactions with measures of white matter microstructure were also observed, suggesting that white matter development may be differentially sensitive to maternal depression and anxiety symptoms in males and females during the prenatal period.

CONCLUSIONS AND RELEVANCE These data highlight the importance of the prenatal period to early brain development and suggest that the underlying white matter microstructure is associated with the continuum of prenatal maternal depression and anxiety symptoms.

\author{
Author Affiliations: Author \\ affiliations are listed at the end of this \\ article. \\ Corresponding Author: Douglas C. \\ Dean III, PhD, Waisman Center, \\ University of Wisconsin, \\ 1500 Highland Ave, \\ Madison, WI 53705 \\ (deaniii@wisc.edu).
}


epression and anxiety are common during pregnancy. ${ }^{1-3}$ These disorders are estimated to affect $7 \%$ to $20 \%$ of pregnant women ${ }^{4}$; however, many women reach only a subclinical threshold ${ }^{1}$ and are not formally diagnosed. Nonetheless, such symptoms pose significant risks not only to mothers but also to their offspring. ${ }^{5,6}$ The sequelae of depression and anxiety symptoms may affect the mother's physiologic responses to distress, ${ }^{7}$ perturbing the intrauterine environment and consequently altering fetal and postnatal development. ${ }^{8,9}$ Mounting evidence links prenatal exposure to maternal depression and anxiety with poorer outcomes in children, including increased negative reactivity, ${ }^{10}$ emotional and behavioral difficulties, ${ }^{11}$ lower verbal IQ, ${ }^{12}$ and physical health problems. ${ }^{13}$ Origins of such maladaptive child outcomes likely have a neurobiological basis, but the extent and timing of neurodevelopmental alterations associated with maternal depression and anxiety symptoms are not well understood.

There is increasing recognition that prenatal maternal stress, depression, and anxiety affect the developing brain, particularly white matter microstructure. White matter is composed of myelin and axons that connect brain regions and facilitate interneuronal communication ${ }^{14}$ and is continuously shaped throughout life. ${ }^{15-17}$ This prolonged maturation makes white matter susceptible to early-life influences, which may have cascading effects on subsequent development. ${ }^{18,19}$ Diffusion tensor imaging (DTI) has been used to examine the association of maternal depression and anxiety with white matter microstructure. ${ }^{2 \mathrm{O}-25}$ Diffusion tensor imaging provides metrics of fractional anisotropy (FA), mean diffusivity (MD), axial diffusivity (AD), and radial diffusivity (RD), which characterize properties of water diffusion in the brain that are sensitive to white matter microstructure features. ${ }^{26,27}$

Nonhuman primate research has found widespread white matter alterations as a result of exposure to prenatal adversity. ${ }^{20-22}$ In humans, increased second-trimester and postpartum maternal depression levels were negatively associated with white matter microstructure in offspring when assessed at preschool ages. ${ }^{23}$ In neonates born to mothers with low and high levels of depression, researchers found lower FA and $\mathrm{AD}$ in the right amygdala, ${ }^{24}$ as well as variations of FA across limbic and prefrontal regions, including the right uncinate and inferior fronto-occipital fasciculi, insular, and parahippocampal white matter. ${ }^{25}$ Collectively, these studies ${ }^{23-25}$ provide converging evidence that white matter may be vulnerable to prenatal maternal adversity. However, it is important to further examine this influence over the continuum of prenatal maternal symptoms, including low and moderate levels. Moreover, in previous studies, ${ }^{23-25}$ DTI was used to characterize brain microstructure. Although informative, DTI lacks microstructural specificity. ${ }^{28,29}$ Techniques such as neurite orientation dispersion and density imaging (NODDI) ${ }^{30}$ may provide improved specificity, allowing new insight into white matter alterations.

To further understand the association between prenatal maternal depression and anxiety symptoms and infant white matter microstructure, we examined mothers with a broad range of symptom severity and performed magnetic

\section{Key Points}

Question Are prenatal maternal depression and anxiety symptoms associated with individual variations of offspring white matter microstructure?

Findings In this cohort study of 101 mother-infant dyads, prenatal maternal depression and anxiety symptoms were associated with infants' white matter microstructure at 1 month of age. Moreover, analyses suggest that prenatal maternal depression and anxiety may be differentially associated with infant male and female microstructure.

Meaning The prenatal environment is important to early brain development, and underlying white matter microstructure may be susceptible to the continuum of maternal depression and anxiety symptom exposure during the prenatal period.

resonance imaging (MRI) on their 1-month-old infants to quantitatively characterize early white matter microstructure. Prenatal maternal symptoms were measured at 28 and 35 weeks' gestation, and infant white matter microstructure was evaluated using DTI and NODDI. ${ }^{30}$ Guided by previous observations that suggest that prenatal exposure to maternal depression and anxiety affects males and females differently, ${ }^{31,32}$ we also investigated associations separately for male and female infants.

\section{Methods}

\section{Study Design and Participants}

Mother-infant dyads $(\mathrm{N}=149)$ participated in sessions as part of an ongoing longitudinal study of the association between early-life experience and infant brain development. Mothers needed to be aged 18 to 40 years, have no diagnosis of psychotic illnesses (eg, schizophrenia, bipolar or borderline personality disorder), and no major autoimmune diseases or infections during pregnancy. Infant inclusionary criteria consisted of uncomplicated singleton birth, no preexisting neurologic conditions or major head trauma, neonatal intensive care unit stay limited to observation only (ie, no interventions), and infant discharged from the hospital with the mother. Criteria were confirmed through interviews with mothers and collected medical records. All study procedures were in accordance with the institutional review board at the University of Wisconsin, Madison. Written informed consent was obtained from each participating family.

\section{Prenatal Maternal Depression and Anxiety Symptoms} Mothers completed the 10-item Edinburgh Postnatal Depression Scale (EPDS) ${ }^{33}$ and the 20-item State-Trait Anxiety Inventory $(\mathrm{STAI})^{34}$ at 28 and 35 weeks' gestation. The state anxiety scale of the STAI was used. The EPDS and STAI measure current levels of symptoms, with higher scores indicative of higher levels of symptoms. Internal consistency for mother selfreported symptoms was high $(a=.86$ at 28 weeks and $a=.84$ at 35 weeks for depression and $a=.85$ at 28 weeks and $a=.84$ at 35 weeks for state anxiety). Depression and state anxiety 
Table 1. Correlation Between 28- and 35-Week Prenatal Maternal Depression and Anxiety Scores

\begin{tabular}{|c|c|c|c|c|c|}
\hline \multirow[b]{3}{*}{ Test } & \multicolumn{5}{|c|}{ Pearson Correlation Coefficient, $r$} \\
\hline & \multicolumn{2}{|l|}{ EPDS } & \multicolumn{2}{|l|}{ STAI } & \multirow{2}{*}{$\begin{array}{l}\text { Composite } \\
\text { Score }\end{array}$} \\
\hline & 28 wk & 35 wk & 28 wk & 35 wk & \\
\hline \multicolumn{6}{|l|}{ EPDS } \\
\hline 28 wk & NA & 0.71 & 0.68 & 0.49 & 0.80 \\
\hline 35 wk & NA & NA & 0.52 & 0.61 & 0.76 \\
\hline \multicolumn{6}{|l|}{ STAI } \\
\hline $28 w k$ & NA & NA & NA & 0.60 & 0.89 \\
\hline 35 wk & NA & NA & NA & NA & 0.84 \\
\hline $\begin{array}{l}\text { Mean (SD) } \\
\text { [range] }\end{array}$ & $5.98(4.13)[0-19]$ & $5.65(3.89)[0-16]$ & $10.68(5.94)[1-34]$ & $11.01(5.48)[2-29]$ & $0.00(8.22)$ \\
\hline
\end{tabular}

Abbreviations: EPDS, Edinburgh Postnatal Depression Scale; NA, not applicable; STAI, State-Trait Anxiety Inventory (state anxiety scale). measures were strongly correlated within and across time (Table 1), and a principal components analysis that incorporated the 4 measures indicated 1 principal component that accounted for $69.5 \%$ of the variance attributed to individual items. This robust depression and anxiety composite was used in subsequent analyses, with higher scores indicative of higher levels of depression and state anxiety symptoms.

\section{MRI Data Acquisition}

Magnetic resonance imaging was performed from October 1, 2014, to November 30, 2016, on a 3-T scanner (MR750 Discovery scanner; General Electric) equipped with a 32-channel head RF array coil (Nova Medical). Scanning occurred at 1 month of age during natural, nonsedated sleep. ${ }^{35}$ As previously described, ${ }^{36,37}$ a MedVac vacuum immobilization bag (CFI Medical Solutions) and foam cushions were used to limit intrascan motion. Noise from the scanner was reduced by derating gradient amplitudes and slew rates and using a soundattenuating bore insert, malleable ear plugs, and neonatal ear covers (MiniMuff; Natus Medical Inc). Electrodynamic headphones (MR Confon) played white noise during image acquisition.

Diffusion-weighted images (DWIs) were acquired using a single-shot, spin-echo. echo-planar imaging pulse sequence. The 10-minute protocol included 69 DWIs, with 9/18/36 diffusion-encoding gradient directions at $\mathrm{b}=350 / 800 / 1500$ seconds $/ \mathrm{mm}^{2}$, respectively, and 6 with no $(\mathrm{b}=0$ seconds/ $\mathrm{mm}^{2}$ ) diffusion weighting. Additional imaging criteria consisted of the following: in-plane resolution, $2 \times 2 \mathrm{~mm}$; slice thickness, $2 \mathrm{~mm}$; repetition time, 8400 milliseconds; echo time, 94 milliseconds; and bandwidth, $3906 \mathrm{~Hz}$ per pixel.

\section{Image Processing}

The DWIs were manually assessed and individual volumes dropped if artifacts were present. The DWIs were coregistered to account for distortion, translation, and rotation from bulk head motion and eddy currents. ${ }^{38}$ Gradient directions were corrected for rotations. ${ }^{39}$ After removal of nonparenchyma signal (ie, 3dSkullStrip ${ }^{40,41}$ ), robust estimation of tensors by outlier rejection ${ }^{42}$ (as part of the diffusion imaging in Python Package ${ }^{43}$ ) estimated the diffusion tensors, and FA, MD, $\mathrm{RD}$, and $\mathrm{AD}$ maps were derived. ${ }^{44,45}$ Each of these tissue criteria reflect quantities sensitive to the underlying microstructure: FA is a scalar quantity between 0 and 1 that describes the degree of diffusion anisotropy, MD reflects total water mobility, $\mathrm{AD}$ describes diffusivity along the main fiber orientation, and RD describes diffusivity perpendicular to the main fiber axis. $^{29,45}$

The NODDI model was also fit to the DWI data. ${ }^{30}$ The volume fraction of the intracellular or restricted diffusion compartment $\left(\mathrm{v}_{\mathrm{IC}}\right)$, the volume fraction of an isotropic diffusion compartment $\left(\mathrm{v}_{\text {ISO }}\right)$, and the orientation dispersion index (ODI) were estimated. Restricted diffusion is attributed to axons and dendrites (neurites); thus, $\mathrm{v}_{\mathrm{IC}}$ is interpreted as an index of neurite density, whereas cerebrospinal fluid or isotropic diffusion is attributed to $\mathrm{v}_{\text {Iso. }}$. The ODI quantifies the angular variation of the neurite orientation (ie, dispersion and fanning) and is sensitive to the degree of fiber coherence. ${ }^{30}$

The participants' FA maps were aligned to a studyspecific template as previously described. ${ }^{36}$ The DTI and NODDI variable maps were brought into study-specific template space by applying the deformations and smoothed with a 5-mm full-width, half-maximum Gaussian filter.

\section{Statistical Analysis}

Statistical analyses used general linear models (GLMs) to test how prenatal maternal adversity (composite of depression and anxiety symptoms) was associated with 1-month infant white matter microstructure. Sex $\times$ symptom interaction and sexspecific GLMs were also used to examine differential associations between prenatal maternal adversity and infant brain in males and females. Voxel-wise GLMs were tested for each DTI (FA, MD, RD, and $\mathrm{AD}$ ) and NODDI ( $\mathrm{v}_{\mathrm{IC}}$ and ODI) quantity and restricted to white matter (eFigure 1 in the Supplement). Multiple comparisons were accounted for by using nonparametric permutation testing (FMRIB Software Library's randomize tool and 10000 permutations) and cluster-based correction, ${ }^{46,47}$ with an initial cluster threshold of $t>2.3$ (approximately $P<.01$ ). Significance was defined as $P<.05$, cluster corrected for familywise error rate.

Infant chronological age (corrected to a 40 -week gestation), maternal educational level, total family income, and breastfeeding status at 1 month were used as covariates to control for potential confounding influences. ${ }^{36,48-51}$ Mean volume-to-volume translation and rotation indexes were computed for each participant ${ }^{52,53}$ and included to ascertain that motion did not confound associations. Infant sex was included as a covariate in analyses of the overall sample. In 
Table 2. Sociodemographic Characteristics of the 101 Mother-Infant Dyads

\begin{tabular}{|c|c|}
\hline Characteristic & Finding $^{\mathrm{a}}$ \\
\hline Mother age at birth, mean (SD), y & $33.22(3.99)$ \\
\hline Mother educational level, mean (SD), y & $17.79(2.45)$ \\
\hline \multicolumn{2}{|l|}{ Total family income, US \$ } \\
\hline $30001-40000$ & $4(4.0)$ \\
\hline $40001-50000$ & $8(7.9)$ \\
\hline $50001-60000$ & $6(5.9)$ \\
\hline $60001-70000$ & $6(5.9)$ \\
\hline $70001-80000$ & $12(11.9)$ \\
\hline $80001-90000$ & $15(14.9)$ \\
\hline $90001-100000$ & $7(6.9)$ \\
\hline $100001-150000$ & $30(29.7)$ \\
\hline $150001-200000$ & $7(6.9)$ \\
\hline$>200000$ & $6(5.9)$ \\
\hline \multicolumn{2}{|l|}{ Mothers' marital status } \\
\hline Married to father & $91(90.1)$ \\
\hline Single & $3(3.0)$ \\
\hline Divorced & $1(1.0)$ \\
\hline Separated & $1(1.0)$ \\
\hline Data not reported or missing & $5(5.0)$ \\
\hline \multicolumn{2}{|l|}{ Mothers' race } \\
\hline African American/black & $2(2.0)$ \\
\hline Asian & $3(3.0)$ \\
\hline White & $92(91.1)$ \\
\hline Native Hawaiian or other Pacific Islander & $2(2.0)$ \\
\hline Mixed race & $1(1.0)$ \\
\hline Not reported & $1(1.0)$ \\
\hline \multicolumn{2}{|l|}{ Mothers' medication status } \\
\hline Pain relief or nonsteroidal & $19(18.8)$ \\
\hline Corticosteroids & $2(2.0)$ \\
\hline Psychotropics & $12(11.9)$ \\
\hline Hormones & $14(13.9)$ \\
\hline None & $54(53.5)$ \\
\hline \multicolumn{2}{|l|}{ Infant sex } \\
\hline Male & $53(52.5)$ \\
\hline Female & $48(47.5)$ \\
\hline Infant age at MRI, mean (range), d & $33.07(18-50)$ \\
\hline Gestation, mean (SD), wk & $39.56(1.40)$ \\
\hline Birth weight, mean (SD), g & $3470(531)$ \\
\hline Birth length, mean (SD), in & $51.38(2.90)$ \\
\hline Head circumference, mean (SD), cm & $13.66(0.57)$ \\
\hline \multicolumn{2}{|l|}{ Apgar score, mean (SD), min } \\
\hline 1 & $8.2(1.4)$ \\
\hline 5 & $8.9(0.6)$ \\
\hline \multicolumn{2}{|l|}{ Breastfeeding status at $1 \mathrm{mo}$} \\
\hline Exclusively breastfed & $87(86.1)$ \\
\hline Exclusively formula fed & $3(3.0)$ \\
\hline Mixed & $11(10.9)$ \\
\hline
\end{tabular}

Abbreviation: MRI, magnetic resonance imaging.

a Data are presented as number (percentage) of mothers or infants unless otherwise indicated.

separate analyses, prenatal maternal medication use was not observed to alter associations with diffusion measures and therefore was not included as a covariate. Other variables, such as birth weight and length and head circumference at birth, are also not related to diffusion measures from this cohort $^{36}$ and thus were not included.

\section{Results}

Of the 149 possible mother-infant dyads, data were missing for 46 infants, who did not sleep through the entire diffusion scan, and 2 mothers, who did not complete all 4 adversity measures at 28 and 35 weeks (eTable in the Supplement provides demographic information of mothers and infants with missing data), resulting in an analysis sample of 101 mother-infant pairs (mean [SD] age of mothers, 33.22 [3.99] years; mean age of infants at MRI, 33.07 days [range, 18-50 days]; 92 white mothers [91.1\%]; 53 male infants [52.5\%]) with successful DWI data and EPDS and STAI reports. Table 2 provides demographic information for mothers and their infants.

The mean (SD) EPDS and STAI scores are reported in Table 1. The sample reported minimal to moderate levels of depression and anxiety, ${ }^{54}$ with 54 mothers (53.5\%) reporting minimal depressive symptoms (EPDS scores, 0-6), 42 mothers (41.6\%) meeting criteria for mild symptoms (EPDS scores, 7-13), and 5 mothers (5.0\%) meeting criteria for moderate depression symptoms (EPDS scores, 14-19). Six mothers reported EPDS scores greater than 12 , which is generally consistent with a diagnosis of major depressive disorder. ${ }^{33}$ Similarly, mothers reported low to medium anxiety symptoms, with STAI scores ranging from a mean of 4 to 28 of a possible range of 0 to 60 . The EPDS and STAI scores were correlated within and across time (Table 1).

\section{Association Between Maternal Depression and Anxiety Symptoms and White Matter Microstructure}

Positive associations were observed between the maternal depression and anxiety symptoms composite and MD, RD, and $\mathrm{AD}$ across white matter regions. Clusters were localized to the right hemisphere and included the anterior, superior, and posterior corona radiata, external capsule, and white matter underlying the dorsolateral prefrontal cortex (Figure 1). More maternal depression and anxiety symptoms were also associated with higher right-hemisphere RD in the superior longitudinal fasciculus and optic radiations. Negative associations between prenatal maternal symptoms and FA were observed, including the external capsule, anterior internal capsule, superior corona radiata, and white matter of the superior-parietal and prefrontal cortices (eFigure 2 in the Supplement). The associations were not observed to be significant based on the specified $P<.05$ cluster-corrected criteria but were suggestive of an association at the $P<.10$ cluster-corrected level.

Maternal depression and anxiety symptoms were also associated with decreased $\mathrm{v}_{\mathrm{IC}}$, particularly in frontal brain regions (Figure 2). Associations were stronger for maternal symptoms in the right hemisphere and included white matter of the superior, middle, and inferior frontal gyrus and the anterior and superior corona radiata. Higher maternal symptoms were additionally associated with lower $\mathrm{v}_{\text {IC }}$ in the righthemisphere external capsule and bilateral white matter of the 
frontolateral orbital gyrus and precentral and postcentral gyrus. No associations were observed with ODI across the whole group.

\section{Association of Infant Sex With Maternal Symptoms and Brain Microstructure}

Analyses further examined whether prenatal maternal depression and anxiety was differentially associated with infant microstructure in males and females. A significant sex $\times$ symptom interaction was found for $\mathrm{FA}$ in regions including the corona radiata, posterior internal capsule, sagittal stratum, and stria terminalis (Figure 3A). A sex $\times$ symptom interaction was also found for $\mathrm{V}_{\text {IC }}$ (Figure 3B) and ODI (Figure 3C) in the sagittal stratum, posterior thalamic radiations, and white matter adjacent to the hippocampus. These results suggest differing associations between males and females, with lower FA and $\mathrm{v}_{\mathrm{IC}}$ in females and higher FA and $\mathrm{v}_{\mathrm{IC}}$ in males exposed to higher levels of maternal depression and anxiety. Separate analyses of the DTI and NODDI measures in males and females revealed several notable associations with maternal symptoms across differential white matter regions (eFigure 3 in the Supplement); however, these associations did not remain significant after accounting for multiple comparisons.

\section{Discussion}

The prenatal period is a critical phase of neurodevelopment, with much of the brain's foundational infrastructure developing during this time. ${ }^{16,17}$ This period is also susceptible to genetic and environmental factors that can alter the offspring's lifelong trajectory of brain development. We provide evidence that prenatal exposure to maternal depression and anxiety symptoms is associated with an infant's white matter microstructure assessed at 1 month. Moreover, although we observed significant alterations across infants, the associations between pre- natal symptoms and white matter were different in male and female offspring. Our findings complement increasing literature that draws attention to the importance of the prenatal period for early brain development ${ }^{19,55}$ and suggest that the infant neural microstructure may be sensitive to prenatal maternal depression and anxiety symptoms.

Prenatal maternal depression and anxiety symptoms were associated with higher DTI diffusivities (MD, RD, and AD) and lower $\mathrm{v}_{\mathrm{IC}}$. These findings are consistent with previous

Figure 1. Association Between Prenatal Maternal Depression and Anxiety Symptoms and Infant Brain Diffusivity

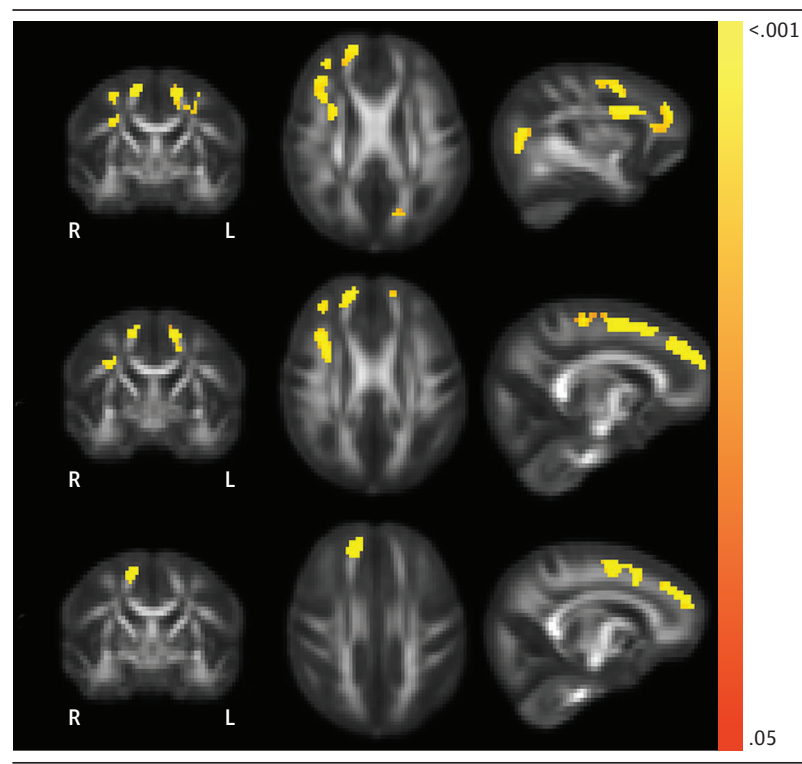

Magnetic resonance imaging shows voxelwise associations between prenatal maternal depression and anxiety composite and radial diffusivity (RD) (top), mean diffusivity (MD) (middle), and axial diffusivity (AD) (bottom), with increased RD, MD, and $A D$ in right-hemispheric white matter. Scale indicates cluster-corrected $P$ values.

Figure 2. Association of Increased Prenatal Maternal Depression and Anxiety Symptoms With Decreased Infant Neurite Density

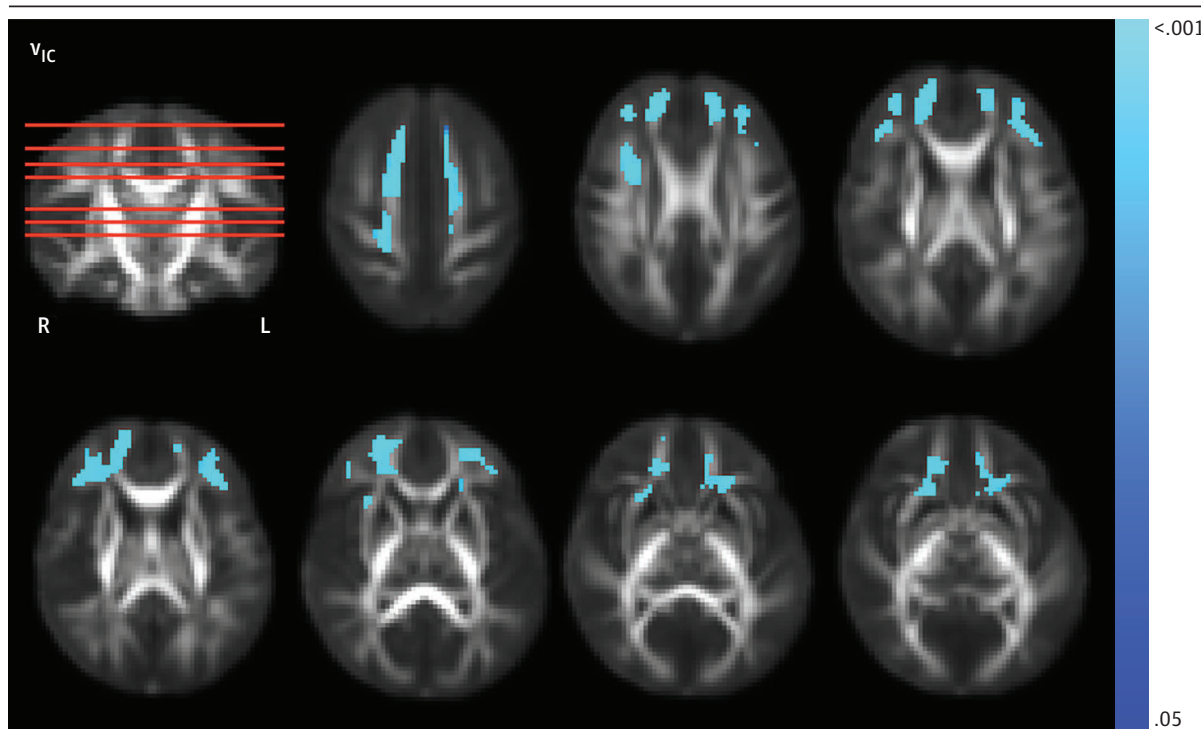

Neurite orientation dispersion and density imaging intracellular volume fraction revealed white matter-spanning bilateral regions of the prefrontal cortex, including white matter of the superior, middle, and inferior frontal gyrus and the anterior and superior corona radiata, that was negatively associated with the prenatal maternal depression and anxiety symptom composite. $\mathrm{V}_{\mathrm{IC}}$ indicates intracellular volume fraction. Scale indicates cluster-corrected $P$ values. 

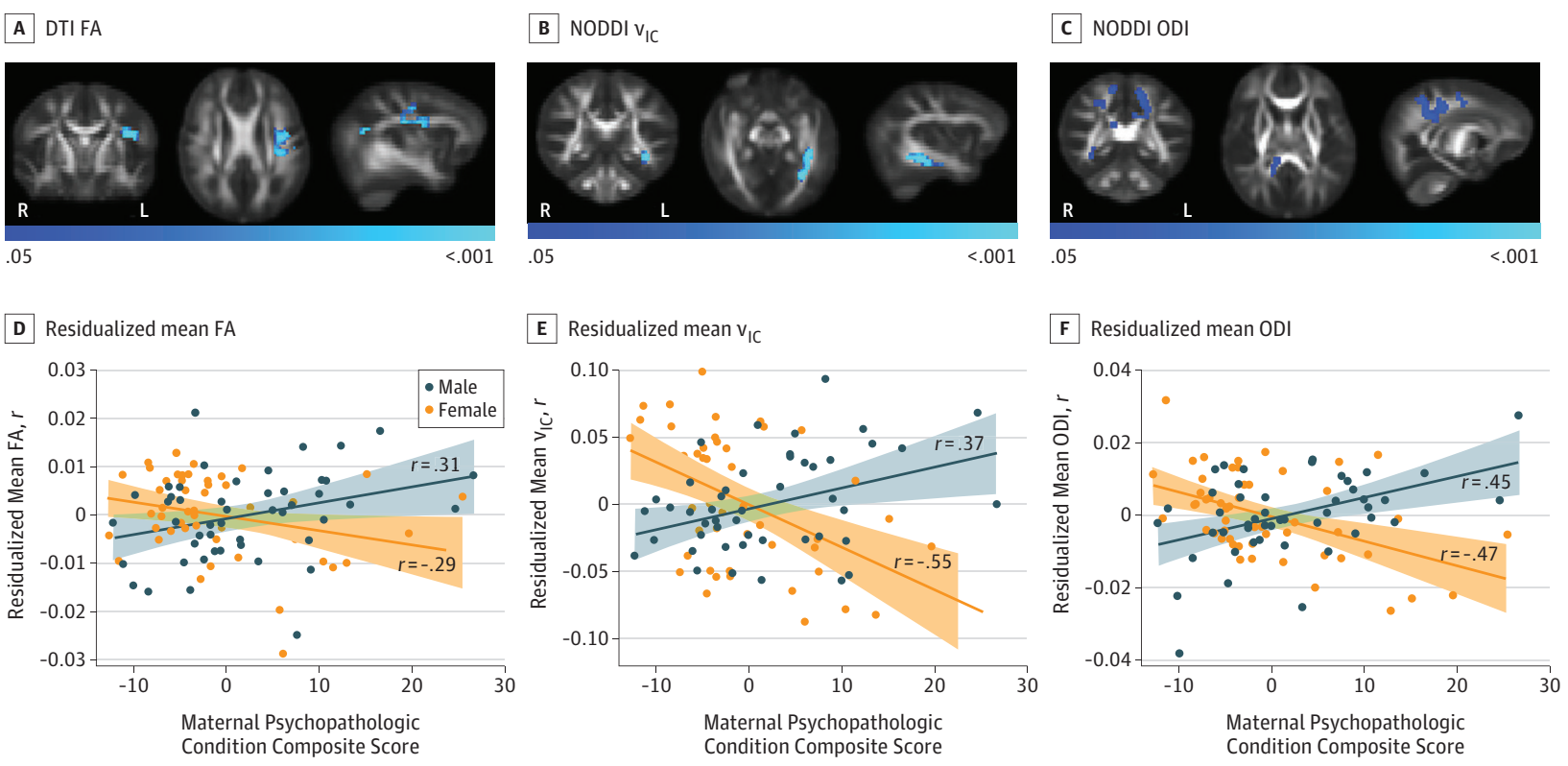

Fractional anisotropy (FA) ( $A$ and $D)$, neurite orientation dispersion and density imaging (NODDI) intracellular volume fraction $\left(\mathrm{V}_{\mathrm{IC}}\right)(\mathrm{B}$ and $\mathrm{E})$, and orientation dispersion index (ODI) ( $\mathrm{C}$ and $\mathrm{F}$ ) were observed to be differentially associated with prenatal maternal adversity in the white matter of males and females, with associations localized within left-hemisphere white matter, including superior-frontal white matter, corona radiata, and splenium of the corpus

studies $^{23-25}$ that examined associations between maternal depressive symptoms and white matter. The nonsignificant negative associations between FA and maternal depression and anxiety that we observed are consistent with results from the only other study ${ }^{25}$ to date that has examined similar associations in neonates.

Conversely, our findings differ from those of Lebel et al, ${ }^{23}$ who found a negative association between maternal depressive symptoms and DTI diffusivity measures in preschool-aged children. This discrepancy may be attributable to the age differences between the sample in our study and the sample of Lebel et al, ${ }^{23}$ highlighting the potential importance of developmental changes on these associations. Future longitudinal studies are necessary to understand the influence of prenatal maternal adversity exposure across differing developmental stages.

Higher diffusivity (MD, RD, and AD) and lower FA and $\mathrm{v}_{\mathrm{IC}}$ are generally assumed to represent poorer white matter microstructure ${ }^{26,29}$ and may reflect disrupted white matter development. ${ }^{17}$ Although our findings are based on associations, they suggest that prenatal maternal symptoms may disrupt white matter development in neonates and potentially have a long-lasting bearing on child development. In particular, many of the white matter regions that were altered are critical for establishing connectivity within brain regions implicated in executive function, cognitive control, and emotion regulation. ${ }^{56}$ Maternal depression and anxiety are known to adversely affect child behavioral and emotional outcomes ${ }^{11}$; our findings show that neural pathways may differ in these children as well. Because of the rapid postnatal maturation of white callosum. Representative plots depict this differing association between males and females, with scatterpoints corresponding to individual mean criteria (FA, $\mathrm{V}_{\mathrm{IC}}$, and $\mathrm{ODI}$ ) and the solid lines corresponding to the lines of best fit with prenatal maternal depression and anxiety symptoms composite. DTI indicates diffusion tensor imaging.

matter ${ }^{16,17,57-61}$ and the likelihood that prenatal and postnatal influences within the first month contribute to shaping brain connectivity, it is likely that any developmental outcome that we study is the joint product of prenatal and postnatal influences. With sufficiently large sample sizes, it will be possible to track neonates and toddlers who may be exposed to prenatal maternal depression and anxiety symptoms at different developmental stages to determine whether prenatal exposure contributes additional variance to subsequent vulnerability above that contributed by postnatal influences.

We also found differing associations of prenatal maternal depression and anxiety symptoms with white matter microstructure in males and females. Such associations may be linked to differing time courses of white matter development, with white matter in females developing earlier than in males. ${ }^{59,62,63}$ The sex differences that we found are consistent with our earlier work showing that early-life stress and cortisol are associated with altered connectivity relations in females but not males. ${ }^{64}$ Moreover, Wen et $\mathrm{al}^{65}$ found the volume and FA of the right amygdala to be positively correlated with maternal depressive symptoms in 4.5-year-old female participants but not in male participants. These findings do not imply that white matter in males is resilient to prenatal maternal symptoms; instead, they suggest the possibility that such microstructural alterations in males may be detected at a different time during development. Whether the sex differences observed in our study are associated with subsequent development of sex differences in the prevalence of depression ${ }^{66}$ requires further longitudinal study. 
The neural mechanisms that underlie associations between microstructural measures and maternal symptoms remain unclear. Axonal formation and growth of long-distance white matter bundles occur almost completely during the second trimester of pregnancy, ${ }^{17}$ whereas myelination begins during the second trimester of pregnancy and continues throughout adolescence and early adulthood. ${ }^{15,17}$ However, prenatal exposure to maternal depression and anxiety may affect each of these processes. Because diffusion measures are sensitive to a broad range of microstructural features, ${ }^{28,29,67}$ future work should consider using techniques that are sensitive to specific tissue properties. ${ }^{45}$

\section{Limitations}

This study has several limitations. First, although our findings suggest a possible association between prenatal maternal adversity and infant white matter, our study design limits our ability to distinguish environmental and genetic influences. Differentiation of such influences may be possible in studies of infant twins. Second, we recognize the difficultly of distinguishing prenatal and postnatal environmental influences. Although we acquired neuroimaging data at 1 month and controlled for factors that shape postnatal neurodevelopment, ${ }^{36,48-51}$ postnatal influences have already begun to affect development by 1 month. Third, the cross-sectional nature of our data restricts our ability to examine trajectories of infant brain development in association with prenatal maternal depression and anxiety symptoms. The current data allow us to establish a 1-month time point from which we can track the influence of later postna- tal experiences. Furthermore, other distal factors may also be related to maternal depression and anxiety, ${ }^{4,68-70}$ which may in turn affect early brain development. ${ }^{50,71-73}$

Experiential factors are important influences that shape early brain development. Our results complement existing literature that reports an association between prenatal depression and anxiety with offspring brain development, specifically white matter microstructure. However, we cannot conclude that the association between prenatal depression and anxiety is directly related in our study; that is, the crossgenerational association could conceivably be genetically mediated. Because our sample was recruited from the community, our findings have important implications for a broader population of mothers than clinic-based samples. The fact that subclinical symptoms predict alterations in early brain development underscores the importance of early intervention even in mothers experiencing mild depression and anxiety symptoms during pregnancy.

\section{Conclusions}

These findings draw attention to the importance of the prenatal period in early brain development. If replicated, our findings also invite more mechanistic studies of early processes that underlie the development of child behavior problems. More speculatively, they also might provide a rationale for the possibility that treatment of maternal depression and anxiety during pregnancy could mitigate how maternal adversity affects infant brain development.

\section{ARTICLE INFORMATION}

Accepted for Publication: May 23, 2018.

Published Online: August 27, 2018 doi:10.1001/jamapediatrics.2018.2132

Author Affiliations: Waisman Center, University of Wisconsin, Madison (Dean, Planalp, Kecskemeti, Adluru, C. K. Schmidt, N. L. Schmidt, Kalin, Goldsmith, Alexander, Davidson); Department of Psychology, University of Wisconsin, Madison (Planalp, Kalin, Goldsmith, Davidson); Center for Healthy Minds, University of Wisconsin, Madison (Wooten, C. K. Schmidt, Frye, Burghy, Short, Davidson); Department of Medical Physics, University of Wisconsin School of Medicine and Public Health, Madison (Birn, Alexander); Department of Psychiatry, University of Wisconsin School of Medicine and Public Health, Madison (Birn, Kalin, Alexander, Davidson); Department of Psychiatry, University of North Carolina at Chapel Hill, Chapel Hill (Styner); Department of Computer Science, University of North Carolina at Chapel Hill, Chapel Hill (Styner)

Author Contributions: Dr Dean had full access to all the data in the study and takes responsibility for the integrity of the data and the accuracy of the data analysis.

Concept and design: Dean, Planalp, Frye, Burghy, N. L. Schmidt, Kalin, Goldsmith, Alexander, Davidson. Acquisition, analysis, or interpretation of data: Dean, Planalp, Wooten, Kecskemeti, Adluru, C. K. Schmidt, Birn, Burghy, N. L. Schmidt, Styner, Short, Kalin, Alexander, Davidson.
Drafting of the manuscript: Dean, Planalp, Wooten, Kecskemeti, Adluru, N. L. Schmidt, Davidson. Critical revision of the manuscript for important intellectual content: Dean, Planalp, C. K. Schmidt Frye, Birn, Burghy, Styner, Short, Kalin, Goldsmith, Alexander, Davidson.

Statistical analysis: Dean, Planalp.

Obtained funding: Burghy, Kalin, Goldsmith, Alexander, Davidson.

Administrative, technical, or material support: Dean Planalp, Wooten, Kecskemeti, Frye, Birn, Burghy, N. Schmidt, Styner, Alexander, Davidson.

Supervision: Adluru, Burghy, Short, Kalin, Goldsmith, Alexander, Davidson.

Conflict of Interest Disclosures: Dr Kalin reported receiving honoraria from CME Outfitters, Elsevier, and the Pritzker Neuropsychiatric Disorders Research Consortium; serving on the advisory boards for Corcept Therapeutics and Skyland Trail-George West Mental Health Foundation; and being a stockholder in Corcept Therapeutics and owning the following patents: promoter sequences for corticotropin-releasing factor alpha (US patent 7071323, issued on July, 4, 2006), a method of identifying agents that alter the activity of the promoter sequences (US patent 7531356 , issued on May 12, 2009), promoter sequences for urocortin II and the use thereof (US patent 7087385, issued on August 8,2006 ), and promoter sequences for corticotropin-releasing factor binding protein and use thereof (US patent 7122650, issued on October 17, 2006). Dr Davidson reported being the founder and president and serving on the board of directors for the nonprofit organization Healthy Minds Innovations Inc and serving on the board of directors for the Mind \& Life Institute. No other disclosures were reported.

Funding/Support: This work was supported by grants P50 MH100031 (Dr Davidson), R01 MH101504 (Dr Goldsmith), K99 MH11059 (Dr Dean), T32 MH018931-26 (Dr Planalp), and K01 MH113710 (Dr Planalp) from the National Institute of Mental Health, National Institutes of Health. Infrastructure support was also provided, in part, by grant U54 HD090256 from the Eunice Kennedy

Shriver National Institute of Child Health and Human Development, National Institutes of Health (to the Waisman Center).

Role of the Funder/Sponsor: The funding sources had no role in the design and conduct of the study: collection, management, analysis, or interpretation of the data; preparation, review, or approval of the manuscript; and decision to submit the manuscript for publication

Disclaimer: The content is solely the responsibility of the authors and does not necessarily represent the official views of the National Institutes of Health.

\section{REFERENCES}

1. Bennett HA, Einarson A, Taddio A, Koren G, Einarson TR. Prevalence of depression during pregnancy: systematic review. Obstet Gynecol. 2004;103(4):698-709. doi:10.1097/01.AOG $.0000116689 .75396 .5 f$ 
2. Evans J, Melotti R, Heron J, et al. The timing of maternal depressive symptoms and child cognitive development: a longitudinal study. J Child Psychol Psychiatry. 2012;53(6):632-640. doi:10.1111/j.1469 -7610.2011.02513.x

3. Heron J, O'Connor TG, Evans J, Golding J, Glover V; ALSPAC Study Team. The course of anxiety and depression through pregnancy and the postpartum in a community sample. J Affect Disord. 2004;80 (1):65-73. doi:10.1016/j.jad.2003.08.004

4. Biaggi $A$, Conroy S, Pawlby S, Pariante CM. Identifying the women at risk of antenatal anxiety and depression: a systematic review. J Affect Disord. 2016;191:62-77. doi:10.1016/j.jad.2015.11.014

5. Kinsella MT, Monk C. Impact of maternal stress, depression and anxiety on fetal neurobehavioral development. Clin Obstet Gynecol. 2009;52(3): 425-440. doi:10.1097/GRF.0b013e3181b52df1

6. Monk C, Spicer J, Champagne FA. Linking prenatal maternal adversity to developmental outcomes in infants: the role of epigenetic pathways. Dev Psychopathol. 2012;24(4):1361-1376 doi:10.1017/S0954579412000764

7. Charil A, Laplante DP, Vaillancourt C, King S. Prenatal stress and brain development. Brain Res Rev. 2010;65(1):56-79. doi:10.1016/j.brainresrev.2010.06 .002

8. O'Donnell K, O'Connor TG, Glover V. Prenatal stress and neurodevelopment of the child: focus on the HPA axis and role of the placenta. Dev Neurosci. 2009;31(4):285-292. doi:10.1159/000216539

9. Seckl JR, Holmes MC. Mechanisms of disease: glucocorticoids, their placental metabolism and fetal 'programming' of adult pathophysiology. Nat Clin Pract Endocrinol Metab. 2007;3(6):479-488. doi:10.1038/ncpendmet0515

10. Davis EP, Snidman N, Wadhwa PD, Glynn LM Schetter CD, Sandman CA. Prenatal maternal anxiety and depression predict negative behavioral reactivity in infancy. Infancy. 2004;6(3):319-331. doi:10.1207/s15327078in0603_1

11. Goodman SH, Rouse MH, Connell AM, Broth MR, Hall CM, Heyward D. Maternal depression and child psychopathology: a meta-analytic review. Clin Child Fam Psychol Rev. 2011;14(1):1-27. doi:10.1007 /s10567-010-0080-1

12. Barker ED, Jaffee $S R$, Uher $R$, Maughan $B$. The contribution of prenatal and postnatal maternal anxiety and depression to child maladjustment. Depress Anxiety. 2011;28(8):696-702. doi:10.1002 /da.20856

13. Avan $B$, Richter $L M$, Ramchandani $P G$, Norris SA, Stein A. Maternal postnatal depression and children's growth and behaviour during the early years of life: exploring the interaction between physical and mental health. Arch Dis Child. 2010;95 (9):690-695. doi:10.1136/adc.2009.164848

14. Fields RD. White matter in learning, cognition and psychiatric disorders. Trends Neurosci. 2008;31 (7):361-370. doi:10.1016/j.tins.2008.04.001

15. Fields RD. White matter matters. Sci Am. 2008; 298(3):42-49.

16. Lebel C, Deoni S. The development of brain white matter microstructure [published online January 3, 2018]. Neuroimage.doi:10.1016/j .neuroimage.2017.12.097

17. Dubois J, Dehaene-Lambertz G, Kulikova S, Poupon C, Hüppi PS, Hertz-Pannier L. The early development of brain white matter: a review of imaging studies in fetuses, newborns and infants. Neuroscience. 2014;276:48-71. doi:10.1016/j .neuroscience.2013.12.044

\section{Andersen SL. Trajectories of brain} development: point of vulnerability or window of opportunity? Neurosci Biobehav Rev. 2003;27(1-2): 3-18. doi:10.1016/S0149-7634(03)00005-8

19. Davidson RJ, McEwen BS. Social influences on neuroplasticity: stress and interventions to promote well-being. Nat Neurosci. 2012;15(5) 689-695. doi:10.1038/nn.3093

20. Coe CL, Lulbach GR, Schneider ML. Prenatal disturbance alters the size of the corpus callosum in young monkeys. Dev Psychobiol. 2002;41(2):178-185. doi:10.1002/dev.10063

21. Coplan JD, Abdallah CG, Tang CY, et al. The role of early life stress in development of the anterior limb of the internal capsule in nonhuman primates. Neurosci Lett. 2010;480(2):93-96. doi:10.1016/j .neulet.2010.06.012

22. Howell BR, McCormack KM, Grand AP, et al. Brain white matter microstructure alterations in adolescent rhesus monkeys exposed to early life stress: associations with high cortisol during infancy. Biol Mood Anxiety Disord. 2013;3(1):21. doi:10.1186/2045-5380-3-21

23. Lebel C, Walton M, Letourneau N, Giesbrecht GF, Kaplan BJ, Dewey D. Prepartum and postpartum maternal depressive symptoms are related to children's brain structure in preschool. Biol Psychiatry. 2016;80(11):859-868. doi:10.1016/j .biopsych.2015.12.004

24. Rifkin-Graboi A, Bai J, Chen $\mathrm{H}$, et al. Prenatal maternal depression associates with microstructure of right amygdala in neonates at birth. Biol Psychiatry. 2013;74(11):837-844. doi:10.1016/j.biopsych.2013.06 .019

25. Rifkin-Graboi A, Meaney MJ, Chen H, et al. Antenatal maternal anxiety predicts variations in neural structures implicated in anxiety disorders in newborns. J Am Acad Child Adolesc Psychiatry. 2015;54(4):313-321.

26. Basser PJ, Jones DK. Diffusion-tensor MRI: theory, experimental design and data analysis: a technical review. NMR Biomed. 2002;15(7-8): 456-467. doi:10.1002/nbm.783

27. Alexander AL, Lee JE, Lazar M, Field AS. Diffusion tensor imaging of the brain. Neurotherapeutics. 2007:4(3):316-329. doi:10.1016/j nurt.2007.05.011

28. Jones DK, Cercignani M. Twenty-five pitfalls in the analysis of diffusion MRI data. NMR Biomed. 2010;23(7):803-820. doi:10.1002/nbm.1543

29. Jones DK, Knösche TR, Turner R. White matter integrity, fiber count, and other fallacies: the do's and don'ts of diffusion MRI. Neuroimage. 2013;73 239-254. doi:10.1016/j.neuroimage.2012.06.081

30. Zhang $\mathrm{H}$, Schneider $\mathrm{T}$, Wheeler-Kingshott $\mathrm{CA}$, Alexander DC. NODDI: practical in vivo neurite orientation dispersion and density imaging of the human brain. Neuroimage. 2012;61(4):1000-1016. doi:10.1016/j.neuroimage.2012.03.072

31. Zohar I, Weinstock M. Differential effect of prenatal stress on the expression of corticotrophin-releasing hormone and its receptors in the hypothalamus and amygdala in male and female rats. J Neuroendocrinol. 2011;23(4):320-328. doi:10.1111/j.1365-2826.2011.02117.x
32. Bale TL, Epperson CN. Sex as a biological variable: who, what, when, why, and how. Neuropsychopharmacology. 2017;42(2):386-396. doi:10.1038/npp.2016.215

33. Cox JL, Holden JM, Sagovsky R. Detection of postnatal depression: development of the 10-item Edinburgh Postnatal Depression Scale. Br J Psychiatry. 1987;150:782-786. doi:10.1192/bjp.150.6.782

34. Spielberger CD. State-Trait Anxiety Inventory. The Corsini Encyclopedia of Psychology. New York, NY: John Wiley \& Sons, Inc; 2010.

35. Dean DC III, Dirks H, O'Muircheartaigh J, et al. Pediatric neuroimaging using magnetic resonance imaging during non-sedated sleep. Pediatr Radiol. 2014:44(1):64-72. doi:10.1007/s00247-013-2752-8

36. Dean DC III, Planalp EM, Wooten W, et al. Mapping white matter microstructure in the one month human brain. Sci Rep. 2017;7(1):9759. doi:10 .1038/s41598-017-09915-6

37. Dean DC III, Planalp EM, Wooten W, et al. Investigation of brain structure in the 1-month infant. Brain Struct Funct. 2018;223(4):1953-1970. doi:10.1007/s00429-017-1600-2

38. Jenkinson M, Bannister P, Brady M, Smith S. Improved optimization for the robust and accurate linear registration and motion correction of brain images. Neuroimage. 2002;17(2):825-841. doi:10 .1006/nimg.2002.1132

39. Leemans A, Jones DK. The B-matrix must be rotated when correcting for subject motion in DTI data. Magn Reson Med. 2009;61(6):1336-1349. doi:10.1002/mrm.21890

40. Cox RW. AFNI: what a long strange trip it's been. Neuroimage. 2012;62(2):743-747. doi:10.1016 /j.neuroimage.2011.08.056

41. Cox RW. AFNI: software for analysis and visualization of functional magnetic resonance neuroimages. Comput Biomed Res. 1996;29(3): 162-173. doi:10.1006/cbmr.1996.0014

42. Chang LC, Jones DK, Pierpaoli C. RESTORE: robust estimation of tensors by outlier rejection. Magn Reson Med. 2005;53(5):1088-1095. doi:10 .1002/mrm.20426

43. Garyfallidis E, Brett M, Amirbekian B, et al; Dipy Contributors. Dipy, a library for the analysis of diffusion MRI data. Front Neuroinform. 2014;8:8. doi:10.3389/fninf.2014.00008

44. Basser PJ, Pierpaoli C. Microstructural and physiological features of tissues elucidated by quantitative-diffusion-tensor MRI. J Magn Reson B. 1996;111(3):209-219. doi:10.1006/jmrb.1996.0086

45. Alexander AL, Hurley SA, Samsonov AA, et al. Characterization of cerebral white matter properties using quantitative magnetic resonance imaging stains. Brain Connect. 2011;1(6):423-446. doi:10.1089/brain.2011.0071

46. Worsley KJ, Andermann M, Koulis T, MacDonald D, Evans AC. Detecting changes in nonisotropic images. Hum Brain Mapp. 1999;8(2-3): 98-101. doi:10.1002/(SICI)1097-0193(1999)8:2 /3<98::AID-HBM5>3.0.CO;2-F

47. Friston KJ, Worsley KJ, Frackowiak RS, Mazziotta JC, Evans AC. Assessing the significance of focal activations using their spatial extent. Hum Brain Mapp. 1994;1(3):210-220. doi:10.1002/hbm .460010306

48. Gilmore JH, Lin W, Corouge I, et al. Early postnatal development of corpus callosum and 
corticospinal white matter assessed with quantitative tractography. AJNR Am J Neuroradiol. 2007;28(9):1789-1795. doi:10.3174/ajnr.A0751

49. Broekman BF, Wang C, Li Y, et al; GUSTO Study Group. Gestational age and neonatal brain microstructure in term born infants: a birth cohort study. PLoS One. 2014;9(12):e115229. doi:10.1371 /journal.pone.0115229

50. Deoni SC, Dean DC III, Piryatinsky I, et al. Breastfeeding and early white matter development a cross-sectional study. Neuroimage. 2013;82:77-86. doi:10.1016/j.neuroimage.2013.05.090

51. Noble KG, Houston SM, Brito NH, et al. Family income, parental education and brain structure in children and adolescents. Nat Neurosci. 2015;18(5) 773-778. doi:10.1038/nn.3983

52. Benner T, van der Kouwe AJ, Sorensen AG. Diffusion imaging with prospective motion correction and reacquisition. Magn Reson Med. 2011;66(1):154-167. doi:10.1002/mrm.22837

53. Yendiki A, Koldewyn K, Kakunoori S, Kanwisher $\mathrm{N}$, Fischl B. Spurious group differences due to head motion in a diffusion MRI study. Neuroimage. 2014;88:79-90. doi:10.1016/j.neuroimage.2013.11 027

54. McCabe-Beane JE, Segre LS, Perkhounkova $Y$ Stuart S, O'Hara MW. The identification of severity ranges for the Edinburgh Postnatal Depression Scale. J Reprod Infant Psychol. 2016;34(3):293-303. doi:10.1080/02646838.2016.1141346

55. Buss C, Entringer S, Moog NK, et al. Intergenerational transmission of maternal childhood maltreatment exposure: implications fo fetal brain development. J Am Acad Child Adolesc Psychiatry. 2017;56(5):373-382. doi:10.1016/j.jaac 2017.03 .001

56. Rothbart MK, Sheese BE, Rueda MR, Posne MI. Developing mechanisms of self-regulation in early life. Emot Rev. 2011;3(2):207-213. doi:10.1177 /1754073910387943
57. Deoni SC, Dean DC III, O'Muircheartaigh J, Dirks $\mathrm{H}$, Jerskey BA. Investigating white matter development in infancy and early childhood using myelin water faction and relaxation time mapping. Neuroimage. 2012;63(3):1038-1053. doi:10.1016/] neuroimage.2012.07.037

58. Dean DC III, O'Muircheartaigh J, Dirks H, et al. Mapping an index of the myelin g-ratio in infants using magnetic resonance imaging. Neuroimage. 2016;132:225-237. doi:10.1016/j.neuroimage.2016.02 040

59. Dean DC III, O'Muircheartaigh J, Dirks H, et al. Characterizing longitudinal white matte development during early childhood. Brain Struct Funct. 2015;220(4):1921-1933. doi:10.1007/s00429 -014-0763-3

60. Lebel C, Beaulieu C. Longitudinal development of human brain wiring continues from childhood into adulthood. J Neurosci. 2011;31(30):10937-10947. doi:10.1523/JNEUROSCI.5302-10.2011

61. Lebel C, Gee M, Camicioli R, Wieler M, Martin W, Beaulieu C. Diffusion tensor imaging of white matter tract evolution over the lifespan. Neuroimage. 2012;60(1):340-352. doi:10.1016/j.neuroimage.201 .11 .094

62. Koolschijn PC, Crone EA. Sex differences and structural brain maturation from childhood to early adulthood. Dev Cogn Neurosci. 2013;5:106-118 doi:10.1016/j.dcn.2013.02.003

63. Schmithorst VJ, Holland SK, Dardzinski BJ. Developmental differences in white matter architecture between boys and girls. Hum Brain Mapp. 2008:29(6):696-710. doi:10.1002/hbm.20431

64. Burghy CA, Stodola DE, Ruttle PL, et al. Developmental pathways to amygdala-prefrontal function and internalizing symptoms in adolescence. Nat Neurosci. 2012;15(12):1736-1741 doi:10.1038/nn.3257

65. Wen DJ, Poh JS, Ni SN, et al. Influences of prenatal and postnatal maternal depression on amygdala volume and microstructure in young children. Transl Psychiatry. 2017;7(4):e1103. doi:10 .1038/tp.2017.74

66. Hankin BL, Abramson LY, Moffitt TE, Silva PA McGee R, Angell KE. Development of depression from preadolescence to young adulthood: emerging gender differences in a 10-year longitudinal study. J Abnorm Psychol. 1998;107(1): 128-140. doi:10.1037/0021-843X.107.1.128

67. Beaulieu C. The basis of anisotropic wate diffusion in the nervous system: a technical review. NMR Biomed. 2002;15(7-8):435-455. doi:10.1002 /nbm.782

68. Ystrom E. Breastfeeding cessation and symptoms of anxiety and depression: a longitudinal cohort study. BMC Pregnancy Childbirth. 2012;12:36. doi:10.1186/1471-2393-12-36

69. Newport DJ, Brennan PA, Green P, et al. Maternal depression and medication exposure during pregnancy: comparison of maternal retrospective recall to prospective documentation. BJOG. 2008;115(6):681-688. doi:10.1111/j.1471-0528 .2008.01701.x

70. Newport DJ, Wilcox MM, Stowe ZN. Antidepressants during pregnancy and lactation: defining exposure and treatment issues. Semin Perinatol. 2001;25(3):177-190. doi:10.1053/sper.2001 .24901

71. Short SJ, Lubach GR, Karasin Al, et al. Materna influenza infection during pregnancy impacts postnatal brain development in the rhesus monkey. Biol Psychiatry. 2010;67(10):965-973. doi:10.1016 /j.biopsych.2009.11.026

72. Thompson BL, Levitt $P$, Stanwood GD. Prenatal exposure to drugs: effects on brain development and implications for policy and education. Nat Rev Neurosci. 2009;10(4):303-312. doi:10.1038/nrn2598

73. Hackman DA, Farah MJ. Socioeconomic status and the developing brain. Trends Cogn Sci. 2009;13 (2):65-73. doi:10.1016/j.tics.2008.11.003 